EQUIPE EDITORIAL E AVALIADORES AD HOC ${ }^{1}$

\title{
EDITORA-CHEFE
}

Dra. Greice da Silva Lorenzzetti Andreis

Instituto Federal de Educação, Ciência e Tecnologia do Rio Grande do Sul (IFRS), Campus Caxias do Sul, Caxias do Sul, RS, Brasil

\section{EDITORA ADJUNTA}

Dra. Katia Arcaro

Instituto Federal de Educação, Ciência e Tecnologia do Rio Grande do Sul (IFRS), Campus Caxias do Sul, Caxias do Sul, RS, Brasil

\section{EDITOR EXECUTIVO}

Esp. David Matos Milhomens

Instituto Federal de Educação, Ciência e Tecnologia de Santa Catarina (IFSC), Campus Florianópolis, Florianópolis, SC, Brasil

\section{COMISSÃO EDITORIAL}

\section{Dra. Daiane Scopel Boff}

Instituto Federal de Educação, Ciência e Tecnologia do Rio Grande do Sul (IFRS), Campus Caxias do Sul, Caxias do Sul, RS, Brasil

Dra. Greice da Silva Lorenzzetti Andreis

Instituto Federal de Educação, Ciência e Tecnologia do Rio Grande do Sul (IFRS), Campus Caxias do Sul, Caxias do Sul, RS, Brasil

\section{Dr. Henri Luiz Fuchs}

Instituto Federal de Educação, Ciência e Tecnologia do Rio Grande do Sul (IFRS), Campus Bento Gonçalves, Bento Gonçalves, RS, Brasil

\section{Me. Jeremias Stein Rodriguês}

Instituto Federal de Educação, Ciência e Tecnologia de Santa Catarina (IFSC), Campus Florianópolis, Florianópolis, SC, Brasil

Me. Michelsch João da Silva

Instituto Federal de Educação, Ciência e Tecnologia de Santa Catarina (IFSC), Campus Florianópolis, Florianópolis, SC, Brasil

\footnotetext{
${ }^{1}$ Edição Especial - Dossiê: Resolução de Problemas na Educação Matemática - Artigos apresentados no I Simpósio de Resolução de Problemas na Educação Matemática (I SiRPEM), da Universidade Estadual de Maringá, Maringá, Paraná, realizado nos dias 29 e 30 de julho de 2021.
} 


\title{
COMISSÃO ORGANIZADORA DO I SIMPÓSIO DE RESOLUÇÃO DE PROBLEMAS NA EDUCAÇÃO MATEMÁTICA
}

\author{
Dr. Marcelo Carlos de Proença - Coordenador Geral \\ Universidade Estadual de Maringá (UEM), Campus Maringá, Programa de Pós-Graduação em Educação para a Ciência \\ e a Matemática, Maringá, PR, Brasil \\ Dra. Érika Janine Maia Afonso \\ Faculdade de Engenharia e Inovação Técnico Profissional (FEITEP), Campus Maringá, Maringá, PR, Brasil
}

Me. Luiz Otavio Rodrigues Mendes

Universidade Estadual de Maringá (UEM), Campus Maringá, Programa de Pós-Graduação em Educação para a Ciência e a Matemática, Maringá, PR, Brasil

\section{Dr. Nelson Antonio Pirola}

Universidade Estadual Paulista (UNESP), Campus Bauru, Programa de Pós-Graduação em Educação para a Ciência, Bauru, SP, Brasil

Me. Wilian Barbosa Travassos

Universidade Estadual de Maringá (UEM), Campus Maringá, Programa de Pós-Graduação em Educação para a Ciência e a Matemática, Maringá, PR, Brasil

\section{AVALIADORES AD HOC}

Dra. Andresa Maria Justulin

Universidade Tecnológica Federal do Paraná (UTFPR), Cornélio Procópio, PR, Brasil

Dr. Arthur Gonçalves Machado Júnior

Universidade Federal do Pará (UFPA), Belém, PA, Brasil

Dra. Edilaine Regina dos Santos

Universidade Estadual de Londrina (UEL), Londrina, PR, Brasil

Dra. Emília de Mendonça Rosa Marques

Universidade Estadual Paulista (UNESP), Bauru, SP, Brasil

Dra. Érica Marlucia Leite Pagani

Centro Federal de Educação Tecnológica de Minas Gerais (CEFET), Belo Horizonte, MG, Brasil

Dra. Érika Janine Maia Afonso

Faculdade de Engenharia e Inovação Técnico Profissional (FEITEP), Maringá, PR, Brasil

\section{Dr. Evandro Tortora}

Instituto Federal de Educação, Ciência e Tecnologia de São Paulo (IFSP), Campus Birigui, Campinas, SP, Brasil

Dra. Fabiane Cristina Höpner Noguti

Universidade Federal de Santa Maria (UFSM), Santa Maria, RS, Brasil

ANDREIS, Greice da Silva Lorenzzetti. Equipe Editorial e Avaliadores Ad hoc. REMAT: Revista Eletrônica da Matemática, Bento Gonçalves, RS, v. 7, n. especial, p. e1002, 30 de dezembro de 2021. https://doi.org/10.35819/remat2021v7iespecialid5567. 


\section{Dra. Giovana Pereira Sander}

Universidade do Estado de Minas Gerais (UEMG), Passos, MG, Brasil

Dr. Jader Otavio Dalto
Universidade Tecnológica Federal do Paraná (UTFPR), Cornélio Procópio, PR, Brasil

Dra. Janaína Poffo Possamai

Universidade Regional de Blumenau (FURB), Blumenau, SC, Brasil

Dr. José Luciano Santinho Lima

Instituto Federal Educação, Ciência e Tecnologia de São Paulo (IFSP), São Carlos, SP, Brasil

$$
\text { Dr. José Sávio Bicho }
$$

Universidade Federal do Sul e Sudeste do Pará (UNIFESSPA), Marabá, PA, Brasil

$$
\text { Dra. Josete Leal Dias }
$$

Universidade Federal do Pará (UFPA), Belém, PA, Brasil

Dra. Juliana Silva de Andrade

Escola Estadual Stela Machado, Bauru, SP, Brasil

Dr. Klinger Teodoro Ciríaco

Universidade Federal de São Carlos (UFSCAR), São Carlos, SP, Brasil

Dra. Liliane Ferreira Neves Inglez de Souza

Universidade Paulista (UNIP), União de Limeira, SP, Brasil

Dra. Luciana Vanessa de Almeida Buranello

Instituto Federal de Educação, Ciência e Tecnologia do Sul de Minas (IFSULDEMINAS), Passos, MG, Brasil

Dra. Luciane de Castro Quintiliano

Instituto Federal de Educação, Ciência e Tecnologia do Sul de Minas (IFSULDEMINAS), Três Corações, MG, Brasil

Dra. Maria Elizabete Rambo Kochhann

Universidade Federal do Sul e Sudeste do Pará (UNIFESSPA), Marabá, PA, Brasil

Dr. Narciso das Neves Soares

Universidade Federal do Sul e Sudeste do Pará (UNIFESSPA), Marabá, PA, Brasil

$$
\text { Dr. Nelson Antonio Pirola }
$$

Universidade Estadual Paulista (UNESP), Bauru, SP, Brasil

\section{Dr. Paulo César Oliveira}

Universidade Federal de São Carlos (UFSCAR), Sorocaba, SP, Brasil

Dr. Richael Silva Caetano

Universidade Estadual do Oeste do Paraná (UNIOESTE), Foz do Iguaçu, PR, Brasil

Dra. Thaís Regina Ueno Yamada

Universidade Estadual Paulista (UNESP), Bauru, SP, Brasil 
Dra. Zionice Garbelini Martos Rodrigues

Instituto Federal de Educação, Ciência e Tecnologia de São Paulo (IFSP), Birigui, SP, Brasil

\section{REVISORES DE TEXTO}

\section{Me. Agostinho Luís Agostini}

Instituto Federal de Educação, Ciência e Tecnologia do Rio Grande do Sul (IFRS), Campus Caxias do Sul, Caxias do Sul, RS, Brasil

\section{Dr. Claudionor Ferreira Araújo}

Instituto Federal de Educação, Ciência e Tecnologia de Alagoas (IFAL), Campus Maceió, Maceió, AL, Brasil

\section{Ma. Fernanda Gonçalves Vieira}

Universidade Federal do Rio Grande (FURG), Rio Grande, RS, Brasil

Secretaria Municipal de Educação e Desporto de Pelotas (SMED), Pelotas, RS, Brasil

Grad. Gabriel Salinet Rodrigues

Universidade Federal de Santa Maria (UFSM), Santa Maria, RS, Brasil

Grad. Joana Restelli Ferla

Universidade do Vale do Rio dos Sinos (UNISINOS), São Leopoldo, RS, Brasil

\section{Dr. Jorgemar Teixeira}

Instituto Federal de Educação, Ciência e Tecnologia do Rio Grande do Sul (IFRS), Campus Caxias do Sul, Caxias do Sul, RS, Brasil

Ma. Luciane da Mota Frota

Escola Estadual Monsenhor Gustavo (EEMG), Montes Claros, MG, Brasil

Ma. Manuela Damiani Poletti da Silva

Instituto Federal de Educação, Ciência e Tecnologia do Rio Grande do Sul (IFRS), Campus Caxias do Sul, Caxias do Sul, RS, Brasil

\section{Me. Marden Oliveira Silva}

Centro Federal de Educação Tecnológica de Minas Gerais (CEFET-MG), Belo Horizonte, MG, Brasil

\section{Dra. Maria Rodrigues de Oliveira \\ São Paulo, SP, Brasil}

Esp. Nathalie de Souza Kappke

Universidade Federal do Rio Grande do Sul (UFRGS), Porto Alegre, Brasil

Me. Ruy Martins dos Santos Batista

Instituto Federal de Educação, Ciência e Tecnologia do Tocantins (IFTO), Palmas, TO, Brasil

\section{Ma. Simone dos Santos França}

Universidade Federal de Mato Grosso do Sul (UFMS), Campo Grande, MS, Brasil 
Grad. Tiele dos Santos Kawarlevski

Universidade Federal do Rio Grande do Sul (UFRGS), Porto Alegre, RS, Brasil

Ma. Verônica Wegner

Instituto Federal de Educação, Ciência e Tecnologia do Rio Grande do Sul (IFRS), Campus Sertão, Sertão, RS, Brasil

Me. Victor Gomes Milani

Prefeitura Municipal de Santa Maria (PMSM), Santa Maria, RS, Brasil

Ma. Viviane Cristina de Mattos Battistello

Universidade Feevale (FEEVALE), Novo Hamburgo, RS, Brasil

\section{APOIO TÉCNICO}

Srta. Glauciane Klein Burgiert Padilha

Instituto Federal de Educação, Ciência e Tecnologia do Rio Grande do Sul (IFRS), Bolsista BICTES/IFRS, Campus

Caxias do Sul, Caxias do Sul, RS, Brasil

\section{Sr. Josmar Chilanti Susin}

Instituto Federal de Educação, Ciência e Tecnologia do Rio Grande do Sul (IFRS), Estudante voluntário, Campus

Caxias do Sul, Caxias do Sul, RS, Brasil 